\title{
Time-Periodically Driven Brownian Motion of Rigid Rod in one dimensional space
}

\author{
M. A. Shahzad 1,2 \\ 1 Department of Physics, Hazara University, Pakistan. \\ 2 Dipartimento di Fisica, Università di Camerino, Camerino, Italy
}

(Dated: April 9, 2018)

\begin{abstract}
In this paper we discuss a simple theoretical approach, taken from the theory of stochastic processes to understand the basic phenomenology of protein translocation through a flickering pore. In this theoretical approach we investigate the dynamics of Brownian particle driven by a periodically driving force. This toy model is further extended by considering the Langevin equation with constants drift and time dependent variance. Using the first passage time theory we derived the formalism for probability density function to comprehend the translocation process occurring in the presence of fluctuating environment.
\end{abstract}

Translocation of biomolecule through nanoscale pores is a complex physical process. Study of translocation process is importance for various biological phenomena such as injection of viral DNA, RNA transfer across nuclear pores, and protein transport through membrane nanopores. Translocation process also play an important role to understand the DNA sequencing and sorting [1-10].

Recently, some theoretical and experimental research groups vary the width of the pore during the translocation process. In such translocation process, the dynamical nature of the pore enable the polymer chain to translocate more efficiently as compare to the translocation of polymer in static pore [11-15]. On the experimental side, it has been shown that the translocation of DNA through a nanochannel can be modulated by dynamically changing the cross section of an elastomeric nanochannel device by applying mechanical stress [16-20]. Timedependent driving force are also used in the translocation process [21-25]. There are some biological examples of such fluctuating environment in translocation are the nuclear pore complex, which plays an essential role in nucleocytoplasmic transport in eukaryotes [26]. Moreover, using an alternating electric field (time-dependent driving force) in the nanopore has been implied as a source for DNA sequencing [28]. P. Fanzio, et al., [17] has shown that the DNA translocation process through an elastomeric nanochannel device can be altered by dynamically changing its cross section. The opportunity to dynamically control the nano-channel dimension open up new possibilities to understand the interactions between bio-molecules and nano-channels, such as the dependence of the translocation dynamics on the channel size, and the effects of moving walls [11]. Here we discuss a theoretical model to understand the translocation processes through a vibrating nanopore.

We consider a Brownian rigid rod particle of length $\lambda$ on one space dimension, driven by a time-periodic driving force, $F(t)=F \cos (\omega t+\theta)$, where $F$ is the amplitude of force, $\omega$ is its frequency and $\theta$ is the phase shift. The translocation of such rigid rod is illustrated in Fig. (1). Assuming that the rigid rod moves as a free Brownian particle in space $(U(x)=0)[5]$, the Langevin equation

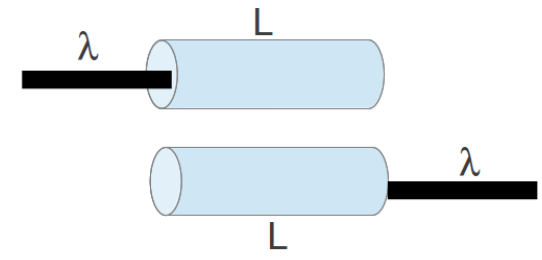

FIG. 1. Translocation of rigid rod particle of length $\lambda$ through a cylinder having length $L$.

for large friction is given by

$$
\frac{d q}{d t}=F \cos (\omega t+\theta)+\sqrt{2 D} \xi(t)
$$

where $\xi(t)$ is a white noise force that has zero mean, $\langle\xi(t)\rangle=0$. The white noise force $\xi(t)$ is delta correlated, $\left.\left\langle\xi(t) \xi\left(t^{\prime}\right)\right\rangle=D \delta\left(t-t^{\prime}\right)\right\rangle$, where $D$ is the diffusion coefficient.

In the limit of low diffusion $D \longrightarrow 0$, the above equation becomes deterministic, that is

$$
\frac{d q}{d t}=F \cos (\omega t+\theta)
$$

Using the initial condition at the edge of channel, $q(0)=$ 0 and $q(\tau)=L$, the integral form of the above equation is

$$
\int_{0}^{L} d q=\int_{0}^{\tau} d t F \cos (\omega t+\theta)
$$

Carrying out the integration, one can easily obtain the formula for the first passage time

$$
\tau(F)=-\frac{\theta}{\omega}+\frac{1}{\omega} \arcsin \left[\frac{\omega L}{F}+\sin (\theta)\right]
$$

Figure (2) shows the plot of the translocation time $\tau(F)$ as a function of constant force $F$ obtained from the above equation. When the force is low enough $(F<10)$, 


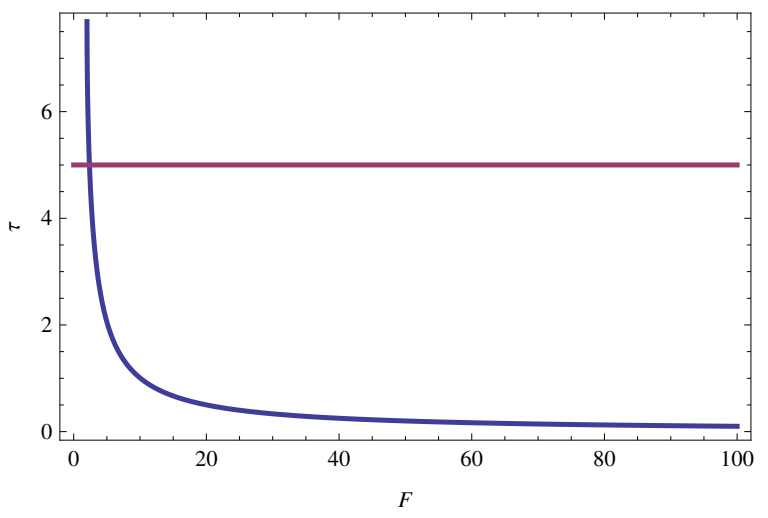

FIG. 2. Translocation time as a function of force $F$ across a channel. The translocation time decrease exponentially with the increase of constant force .

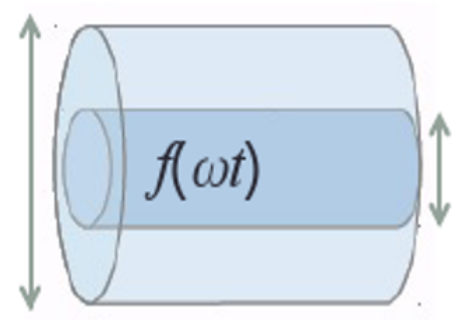

FIG. 3. Fluctuating rigid rod particle of length $\lambda$ around the minima.

there is an abrupt change in time, the time $\tau$ increases with the decrease of the constant force $F$. However, the time becomes insensitive with respect to force change in the limit of $F$ large.

We consider the encounter of the rod-like particle at the pore and its eventual escape into either the cis or trans side as a one-dimensional stochastic process of the particle under the action of applied driven force $F$ across the channel. The equation of motion for the state variable $q(x)$ can be formulated in the form of a stochastic differential equation (Langevin equation) given by

$$
\frac{d q}{d t}=\mu F+\sqrt{2 D} f(t) \xi(t)
$$

where $F$ is constant force, the friction $\mu$ is referred to as the drift coefficient, while $D$ is called the diffusion coefficient, and $\xi(t)$ is a white noise. The fluctuating variance $\sigma(t)^{2}=f(t)=A \cos (\omega t+\theta)$, where $A$ is amplitude, $\omega$ is the frequency of the fluctuating variance. The schematic diagram of the such process are shown in Fig. (3).

Using the initial condition $q(0)$ and the boundary condition $q(\tau)=L+\lambda$, the expression for average first

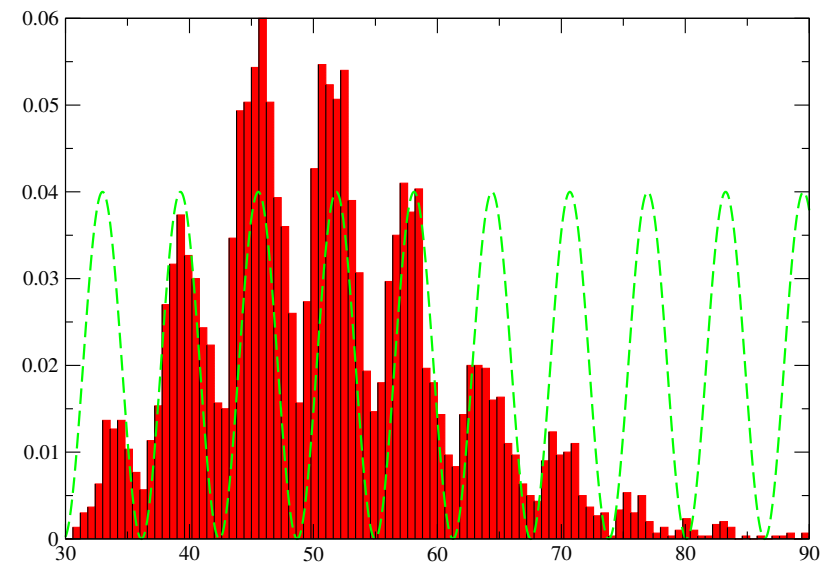

FIG. 4. The distribution of the first passage time at $\omega=5$, $\theta=0$ and $F=1$, computed via numerical simulation of Eq.(5). The distribution displays many peaks locked to the variance modulation, $f(\omega t)=A \cos (\omega t+\theta)$.

passage time can be easily obtained by using Eq. (3.26),

$$
\begin{aligned}
\langle\tau\rangle & =\frac{1}{D} \int_{0}^{L+\lambda} \exp (-F \mu x) d x \int_{x}^{L+\lambda} \exp (F \mu y) d y \\
& =\frac{L+\lambda}{D \mu F}
\end{aligned}
$$

The simplest time-discrete approximation of the Langevin equation is the stochastic generalization of the Euler approximation

$$
\begin{aligned}
Y_{n+1}= & Y_{n}+\mu F \Delta_{n}+\sqrt{2 D \Delta_{n}} \cos (\omega t) \Delta \xi_{n} \\
& n=1, \ldots, N-1
\end{aligned}
$$

with the initial value $Y_{0}=x_{0}$. The time step are $\Delta_{n}=$ $t_{n+1}-t_{n}$, and $\Delta \xi_{n}=\xi\left(t_{n+1}\right)-\xi\left(t_{i}\right)$.

In Fig. (4) we plot the first passage density obtained via direct simulation of Eq. (5). The distribution of the first passage density shows many peaks which are locked to the variance modulation (green dotted line in Fig. (4)). The Langevin equation with time dependent diffusion term $\sigma(t)$ is given by

$$
d q(t)=\mu F d t+\sigma(t) d \xi(t) .
$$

where $q$ is reaction coordinate. A reaction coordinate $q$ defined via the piecewise function [34]

$$
q(x)= \begin{cases}0 & \text { if } x<0 \\ x / L & \text { if } 0 \leq x<L \\ 1 & \text { if } x \geq 1\end{cases}
$$

where $x$ is the coordinate of the particle. Here, $q=0$ when particle is on the cis-side of the channel, $q=1$ when the particle is completely on the trans-side, and $0<q<1$ if there is any particle inside the channel. The expression for collective coordinate becomes

$$
q=\frac{1}{N} \sum_{i=1}^{N} q\left(x_{i}\right) .
$$




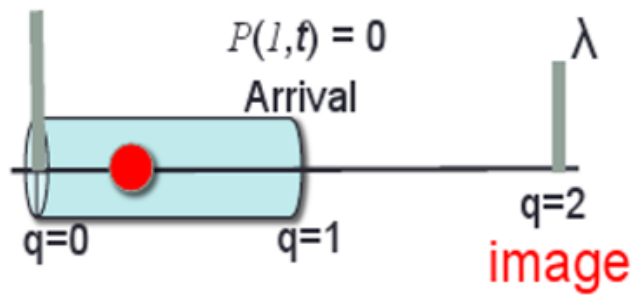

FIG. 5. Schematic figure of the method of images showing the translocation of particle through a channel.

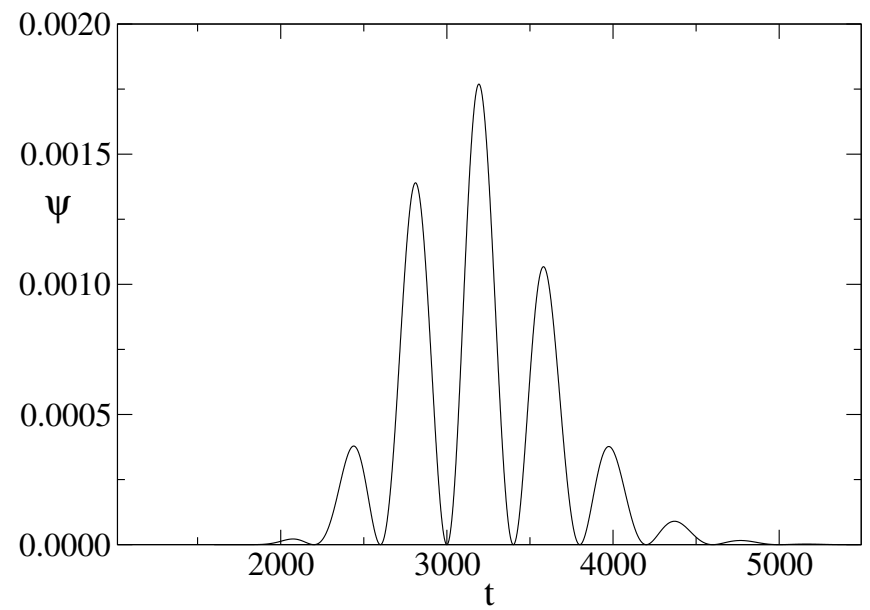

FIG. 6. The distribution of first passage time computed via approximate solution of Langevin equation (Eq. (5)).

The associated Fokker-Planck equation [31, 32] describing the evolution of the probability density function $(\mathrm{PdF})$ of $q(t)$ can be expressed as

$$
\frac{\partial p\left(q, t \mid x_{0}\right)}{\partial t}=-\mu F \frac{\partial p\left(q, t \mid x_{0}\right)}{\partial q}+\frac{1}{2} \sigma^{2}(t) \frac{\partial^{2} p\left(q, t \mid x_{0}\right)}{\partial q^{2}}
$$

where $p\left(q, t \mid x_{0}\right)$ is transition $\mathrm{PdF}$ with initial condition $P(q, 0)=\delta(q)$. Changing the original variable $q$ and $t$ into

$$
\tau(t)=\frac{1}{2} \int \sigma^{2}(t) d t=\frac{A^{2}}{4}\left[t+\frac{1}{2 \omega} \sin (\omega t)\right]
$$

with $\sigma(t)=A \cos (\omega t)$, and

$$
z=q-\int \mu F d t=q-\mu F t
$$

the normalized solution of Fokker Planck equation in the unbounded space becomes,

$$
P\left(q, t \mid x_{0}\right)=\frac{1}{2 \sqrt{\pi \tau(t)}} \exp \left[-\frac{\left(q-q_{0}-\mu F t\right)^{2}}{4 \tau(t)}\right]
$$

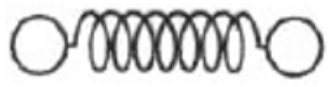

L

FIG. 7. Two particle attached via Hooke's spring translocating through a channel of length $L$.

We used the method of images for solving the boundary value problems with time dependent diffusion coefficient. To solve the FP equation using the method of images, we place a mirror source at $\mathrm{q}=2$ (as shown in Fig. (5)) such that the solution of the FP equation emanating from the original and mirror sources exactly compensate each other at the position of the mirror source at each instant of time. This implies that the initial condition $P(x, 0)=$ $\delta(x)$ must be modified to

$$
P(q, 0)=\delta(q)+\lambda \delta(q-2)
$$

where $\lambda$ determines the strength of the mirror image source. Assuming the initial condition of particle capture into the channel as: $P(q, 0)=\delta(q)$, and the absorbing boundary condition once the particle has completely translocated into trans side of the channel: $P(q=1, t)=$ 0 . From this, the result for first passage probability density function (FP-PdF) [32] can be written as:

$$
\Psi(t)=\frac{L \cos ^{2}(\omega t)}{\sqrt{4 \pi \tau(t)^{3}}} \exp \left[-\frac{(1-\mu F t)^{2}}{4 \tau(t)}\right]
$$

where $\Psi(t)$ describes the probability density for the time that the particle spends in the channel. Figure (6) show the plot of the Eq. (16). The distribution of FPT displays many speaks at frequency $\omega=3$. Note that an increase in the frequency of oscillation $\omega$ causes an increase in the number of peaks in the distribution of first passage time (FPT). From this simple theory of particle translocation through a channel, we predict the peaks in the first passage time PdF when the transport process is perturbed by a simple time modulation of variance.

Using the approximation of absorbing boundary condition at the trans side, $q=L$, the solution of $\Psi(t)$ contains the trajectory of the random walker traveled to $q<0$ side and come back to the region $0<q<L$. During particle translocation, there is a finite probability that the particle can retract into the cis side against the free energy barrier. Under such boundary condition the particle cannot enter the interval through an absorbing end point because its is instantly trapped through by the absorbing boundary.

We anticipate that by introducing a simple time modulation in the variance of Langevin equation, the transport of single particle across a channel display many peaks in the distribution of translocation time. It is instructive 
translocation time data

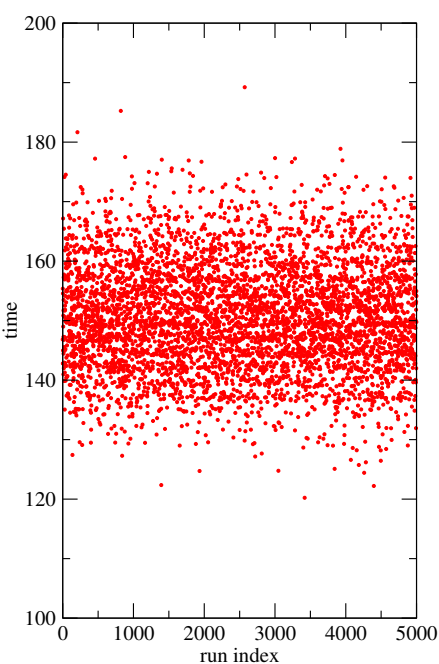

PdF of translocation time

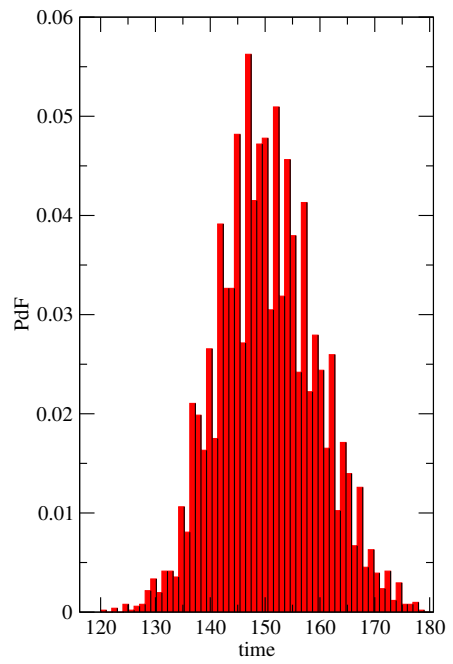

FIG. 8. Right Panel: Probability distribution function of translocation time for two particle attached via Hooke's spring $K=0.5$ at frequency $\omega=3$. Left Panel: The dots represent the scatter data of translocation time.

to investigate the transport dynamics of dumbbell particle in order to understand the interplay of channel frequency and the oscillation of spring. In such toy model the dumbbell are described by harmonic oscillator, composed by two material points and connected via Hooke's spring of elastic constant $k$.

Consider an elastic dumbbell particle made of two identical particle connected by a Hookean spring. The

equation of motion for the particle 1 of a dumbbell is of the Langevin type

$$
\gamma \frac{d x_{1}}{d t}=k\left(x_{2}-x_{1}-a\right)+F_{1}+A f(\omega t) \xi_{1}(t)
$$

and similarly for particle 2

$$
\gamma \frac{d x_{2}}{d t}=-k\left(x_{2}-x_{1}-a\right)+F_{2}+A f(\omega t) \xi_{2}(t)
$$

where $F_{1}$ and $F_{2}$ are constant forces acting on particle 1 and particle 2 , respectively, and $k$ is the Hook spring constant. Figure (7) illustrates the basic scheme of dumbbell particle translocation through a fluctuating channel.

Equations (17) and (18) are solved numerically using the standard Euler-Maruyama method [33]. Figure (8) describes the probability distribution function $(\mathrm{PdF})$ of translocation time.

In summary, we discuss a simple theoretical model to understand the protein translocation through a flickering pore. We study the dynamics of Brownian particle driven by a periodically driving force acting along the axis of the nanopore. Using Langevin equation with constants drift and time dependent variance, and first passage time theory we obtained the formalism for translocation time probability density function $(\mathrm{PdF})$.

The author would like to thank U. M. B. Marconi, A. Vulpian and F. Cecconi for helpful discussion, and Institute of Complex Systems, National Research council of Rome for providing access to computational resources.
[1] Amit Meller, Lucas Nivon, and Daniel Branton. VoltageDriven DNA Translocations through a Nanopore. Phys. Rev. Lett., 86, 3435, 2001.

[2] John J. Kasianowicz, Eric Brandin, Daniel Branton, and David W. Deamer. Characterization of individual polynucleotide molecules using a membrane channel. Proc. Natl. Acad. Sci. USA, 93, 13770:13773, 1996.

[3] Amit Meller, Lucas Nivon, Eric Brandin, Jene Golovchenko, and Daniel Branton. Rapid nanopore discrimination between single polynucleotide molecules. Biochemistry, 97, 1079:1084, 1999.

[4] A. M. Berezhkovskii, M. A. Pustovoit, and S .M. Bezrukov. Channel-facilitated membrane transport: Transit probability and interaction with the channel. Journal of Chemical Physics, 116, 9952:9956, 2002.

[5] A. M. Berezhkovskii and I. V. Gopich. Translocation of Rod-like Polymers through Membrane Channels. Biophys J., 84, 787:793, 2003.

[6] D. K. Lubensky, and D. R. Nelson. Driven polymer translocation through a narrow pore. Biophys. J., 77, 1824:1838, 1999.

[7] N. Lee and and S. Obukhov. Diffusion of a polymer chain through a thin membrane. J. Phys. II., 6, 195:204, 1996.

[8] Muthukumar, M. Polymer translocation through a hole.
J. Chem. Phys., 111, 10371:10374, 1999.

[9] Muthukumar, M. Translocation of a confined polymer through a hole. Phys. Rev. Lett., 86, 3188:3191, 2001

[10] P. Ansalone, M. Chinappi, L. Rondoni, F. Cecconi. Driven diffusion against electrostatic or effective energy barrier across -Hemolysin. J. Chem. Phys., 143, 154109 2015.

[11] J. A. Cohen, Chaudhuri, A. and Golestanian R. Active Polymer Translocation through Flickering Pores. Phys. Rev. Lett., 107, 238102, 2011.

[12] Rowghanian, P.; Grosberg, A.Y. Force-Driven Polymer Translocation through a Nanopore: An Old Problem Revisited. J. Phys. Chem. B, 115, 14127, 2011.

[13] Ikonen, T.; Bhattacharya, A.; Ala-Nissila, T.; Sung,W. Influence of non-universal effects on dynamical scaling in driven polymer translocation. J. Chem. Phys., 137, 085101, 2012.

[14] Adhikari, R.; Bhattacharya, A. Driven translocation of a semi-flexible chain through a nanopore: A Brownian dynamics simulation study in two dimensions. J. Chem. Phys.,138, 204909, 2013.

[15] Dubbeldam, J.L.A.; Rostiashvili, V.G.; Milchev, A.; Vilgis, T.A. Forced translocation of a polymer: Dynamical scaling versus molecular dynamics simulation. Phys. Rev. 
E, 85, 041801, 2012.

[16] H. Zhang, Zhao Q, Tang Z, Liu S, Li Q, Fan Z, Yang F, You L, Li X, Zhang J, Yu D. Slowing down DNA translocation through solid-state nanopores by pressure. Small., 9, 4112:7, 2013.

[17] Paola Fanzio, Chiara Manneschi, Elena Angeli, Valentina Mussi, Giuseppe Firpo, Luca Ceseracciu, Luca Repetto and Ugo Valbusa. Modulating DNA Translocation by a Controlled Deformation of a PDMS Nanochannel Device. Scientific Reports, 2, 791, 2012.

[18] D. Huh, Mills KL, Zhu X, Burns MA, Thouless MD, Takayama S. Tuneable elastomeric nanochannels for nanofluidic manipulation. Nat Mater., 6, 424:8, 2007.

[19] E. Angeli, Manneschi C., Repetto L., Firpo G. and Valbusa U. DNA Manipulation with Elastomeric Nanostructures Fabricated by Soft-Moulding of a FIB-Patterned Stamp. Lab Chip, 11, 2625, 2011.

[20] Nicholas A. W. Bell, Murugappan Muthukumar, Ulrich F. Keyser Translocation frequency of double-stranded DNA through a solid-state nanopore. arXiv:1508.04396 [physics.bio-ph], 2015.

[21] T. Ikonen, J. Shin, W. Sung, T. Ala-Nissila. Polymer translocation under time-dependent driving forces: Resonant activation induced by attractive polymer-pore interactions. The Journal of chemical physics, 136, 205104, 2012.

[22] A. Fiasconaro, J. J. Mazo, and F. Falo. Active polymer translocation in the three-dimensional domain. Phys. Rev. E, 91, 022113, 2015.

[23] Stefureac R. I, Kachayev A, Lee J. S. Modulation of the translocation of peptides through nanopores by the application of an AC electric field. Chem. Commun., 48, 1928:30, 2012.
[24] M. Bates, Burns M, Meller A. Dynamics of DNA molecules in a membrane channel probed by active control techniques. Biophys J., 84, 2366:72, 2003.

[25] Jalal Sarabadani, Timo Ikonen, Tapio Ala-Nissila Theory of polymer translocation through a flickering nanopore under an alternating driving force. arXiv:1505.04057 [cond-mat.stat-mech], 2015.

[26] J. Yamada, J. L. Phillips, S. Patel et al., A bimodal distribution of two distinct categories of intrinsically disordered structures with separate functions in FG nucleoporins. Mol Cell Proteomics., 9, 2205:24, 2010.

[27] P. Rehling, Brandner K, Pfanner N. Mitochondrial import and the twin-pore translocase. Nat. Rev. Mol. Cell Biol., 5, 519:30, 2004.

[28] G. Sigalov, Jeffrey Comer, Gregory Timp, and Aleksei Aksimentiev. Detection of DNA sequences using an alternating electric field in a nanopore capacitor. Nano Lett., 8, 56:63 2008.

[29] Gregory F Schneider and Cees Dekker. DNA sequencing with nanopores. Nature Biotechnology, 30, 326:328, 2012.

[30] Meni Wanunu. Nanopores: A journey towards DNA sequencing. Phys Life Rev. 9, 125:158, 2012.

[31] A. Molini, P. Talkner, G.G. Katul, A. Porporato. First passage time statistics of Brownian motion with purely time dependent drift and diffusion. Physica A, 390, 1841:1852, 2011.

[32] S. Redner. A Guide to First Passage Processes. Cambridge University Press, Cambridge, UK, 2001.

[33] P. E. Kloeden and Platen, E. Numerical Solution of Stochastic Differential Equations. Springer, Berlin, 1992.

[34] J. M. Polson and A. C. McCaffrey. Polymer translocation dynamics in the quasi-static limit. J. Chem. Phys.,138, 174902. 2013, 